\title{
Poliuretana Derivada de Óleos Vegetais Exposta ao Intemperismo Artificial
}

\author{
Alessandra E. F. S. Almeida \\ Escola de Engenharia de São Carlos, USP \\ Osny P. Ferreira \\ Departamento de Arquitetura e Urbanismo, EESC, USP
}

\begin{abstract}
Resumo: Foram obtidas as propriedades mecânicas e viscoelásticas da poliuretana derivada do óleo de mamona após sua exposição ao intemperismo artificial, a fim de avaliar a aplicabilidade deste material como revestimento polimérico para substratos de concreto na Construção Civil. Os procedimentos experimentais foram realizados em conformidade com a ASTM G 53 for "Operating Light and Water Exposure Apparatus (Fluorescent UV - Condensation Type) for Exposure of Nonmetallic Materials" "[1]. O ensaio para a caracterização mecânica dos corpos-de-prova após exposição ao intemperismo artificial foi realizado conforme a norma ASTM D 638M-96 "Standard Test Method for Tensile Properties of Plastics (Metric)"[2]. Foi empregado o método de análise dinâmico-mecânica para a obtenção das propriedades viscoelásticas da poliuretana vegetal. Os resultados mostraram que a exposição ao intemperismo artificial não ocasionou mudanças significativas nas propriedades do revestimento polimérico para o tempo de exposição estudado.
\end{abstract}

Palavras chave: Poliuretana vegetal, propriedades mecânicas, propriedades viscoelásticas, intemperismo artificial.

\section{Polyurethane Derived from Vegetal Oil Exposed to Artificial Weathering}

Abstract: The mechanical and viscoelastic properties of polyurethane derived from castor oil were obtained after being exposed to artificial weathering with the purpose of evaluating their applicability as polymeric coatings on concrete substrate for Civil Construction. The procedures followed the ASTM G53 for "Operating Light and Water Exposure Apparatus (Fluorescent UV - Condensation Type) for Exposure of Nonmetallic Materials" ${ }^{\text {"[] }}$. Mechanical properties were obtained by means of the ASTM D 638M-96 "Standard Test Method for Tensile Properties of Plastics (Metric)"[2]. Furthermore, a Dynamic Mechanical Analysis (DMA) was carried out in order to study the viscoelastic properties of polyurethane. The results showed that the properties were altered slightly considering the time studied.

Keywords: Polyurethane from castor oil, mechanical properties, viscoelastic properties, artificial weathering.

\section{Introdução}

A degradação dos polímeros utilizados em revestimento para proteção das estruturas de concreto é evidenciada, muitas vezes, pelo escurecimento ou amarelecimento superficial e enfraquecimento do filme. A causa das mudanças dessas propriedades pode estar no aparecimento de duplas ligações decorrente da perda de átomos de hidrogênio como radicais, os quais se combinam para produzir hidrogênio gasoso, ou são oxidados para a formação de água.

A radiação ultravioleta é reconhecida como fator degradante de materiais poliméricos, pois está associada ao mecanismo de foto-degradação. A indústria dos revestimentos poliméricos tem alcançado enormes avanços, com o objetivo de facilitar a aplicação e melhorar o desempenho dos sistemas de proteção. Um fator que vem merecendo destaque consiste em reduzir a emissão de materiais volá- teis na atmosfera, existentes nos solventes orgânicos. Atualmente, são aplicados revestimentos $100 \%$ sólidos, ou seja, que não utilizam solventes e suas características são modificadas de acordo com as proporções e propriedades dos elementos constituintes.

A resina poliuretana de origem do óleo de mamona avaliada neste trabalho foi elaborada pelo Laboratório de Química Analítica e Tecnologia dos Polímeros do Instituto de Química de São Carlos/ Universidade de São Paulo, apresentando diversas vantagens, principalmente devido não prejudicar a saúde do manipulador ${ }^{[3]}$. A sua versatilidade como material possibilita sua aplicação como revestimento polimérico na forma de película aplicada a matrizes cimentíceas na construção civil, apresentando elevada resistência química e bom desempenho ${ }^{[4]}$.

Os revestimentos poliméricos são aplicados com o objetivo de impedir o transporte de agentes para o interior do concreto e conservar a sua integridade como material em-

Autor para correspondência: Alessandra E. F. S. Almeida, Escola de Engenharia de São Carlos, USP, Avenida do Trabalhador Saocarlense 400, CEP: 13560-970, São Carlos, SP. E-mail: aefsouza@ig.com.br 
pregado em construções. Entretanto, é importante lembrar que os polímeros expostos às intempéries sofrem a ação da radiação solar combinada com a presença de oxigênio, entre outros elementos, que podem resultar nas modificações de suas propriedades. Assim, neste trabalho foram realizados procedimentos experimentais para a avaliação da resistência ao intemperismo artificial deste sistema polimérico estudado, através da obtenção de suas propriedades mecânicas e viscoelásticas.

O termo degradação de polímeros é empregado para denotar mudanças nas propriedades físicas causadas por processos envolvendo as ligações químicas entre as moléculas ${ }^{[5]}$. A energia radiante pode produzir profundas mudanças em materiais orgânicos, conforme a substância irradiada e a natureza da radiação. A radiação é capaz de atuar no orbital dos elétrons dos átomos e, portanto, nas ligações interatômicas ${ }^{[6]}$. A radiação ultravioleta proveniente do espectro solar tem sua energia aumentada inversamente ao comprimento de onda, e radiações com comprimento de onda próximo de $350 \mathrm{~nm}$ apresentam energia suficiente para quebrar ligações entre os átomos de carbono e produzir radicais. $\mathrm{O}$ efeito da radiação ultravioleta acelera a taxa de oxidação dos polímeros ${ }^{[7]}$.

As principais propriedades dos polímeros dependem da natureza das moléculas constituintes e da força de ligação entre $e^{e l a s}{ }^{[8]}$. Portanto, as propriedades físicas dos polímeros são governadas pela estrutura e tamanho das macromoléculas que determinam as forças intermoleculares. O estudo do comportamento mecânico dos sistemas de proteção poliméricos é importante, pois a estrutura protegida pode sofrer deformações resultando em tensões no revestimento. Foram obtidas as seguintes propriedades mecânicas para os sistemas estudados: resistência à tração, módulo de elasticidade, tensão de escoamento, tensão de ruptura e alongamento na ruptura.

A temperatura de transição vítrea é um parâmetro importante para a caracterização dos polímeros, pois está relacionada com as suas propriedades mecânicas e com a temperatura na qual o revestimento apresentará melhor desempenho. $\mathrm{O}$ material polimérico apresenta-se rígido ou duro para temperaturas inferiores a de transição vítrea, e torna-se flexível ou deformável quando em temperaturas superiores a de transição vítrea. Desse modo, a transição vítrea ocorre num intervalo de temperatura em que o polímero passa do estado vítreo para o viscoso.

Devido ao comportamento viscoelástico dos polímeros, pode-se dizer que eles têm a capacidade de armazenar energia mecânica, como os materiais elásticos, e dissipar energia como os materiais viscosos. Assim, quando o polímero é submetido a uma tensão senoidal oscilatória, a tensão e deformação podem ser descritas $\operatorname{como}^{[9]}$ :

$$
\begin{aligned}
& \sigma=\sigma_{0} \operatorname{sen}(\omega \mathrm{t}+\delta)=\sigma_{0} \operatorname{sen} \omega \mathrm{t} \cos \delta+\sigma_{0} \cos \omega \mathrm{t} \operatorname{sen} \delta \\
& \varepsilon=\varepsilon_{0} \operatorname{sen} \omega \mathrm{t}
\end{aligned}
$$

onde $\sigma$ é a tensão, $\varepsilon$ é a deformação, $\omega$ a freqüência angular, e $\delta$ é o ângulo de fase. Desse modo, a tensão é considerada como tendo um componente em fase com a resposta $\left(\sigma_{0} \cos \delta\right)$ e outro defasado $90^{\circ}\left(\sigma_{0}\right.$ sen $\left.\delta\right)$. Dividindo-se a tensão pela deformação e, tem-se os módulos:

$$
\begin{aligned}
& E^{\prime}=\sigma_{0} \cos \delta / \varepsilon_{0} \\
& E^{\prime \prime}=\sigma_{0} \operatorname{sen} \delta / \varepsilon_{0}
\end{aligned}
$$

A resposta à solicitação pode ser expressa por:

$$
\mathrm{E}^{*}=\mathrm{E}^{\prime}+\mathrm{i} \mathrm{E}^{\prime}
$$

Onde o componente E' é o módulo de armazenamento, relacionado com a energia armazenada em cada ciclo, e E" é o módulo de perda, relacionado com a energia dissipada em forma de calor. A relação:

$$
\tan \delta \frac{E^{\prime \prime}}{E^{\prime}}
$$

representa a razão entre a energia perdida e a armazenada, chamada de fator de perda, ou Tan Delta.

A temperatura de transição vítrea de diferentes sistemas poliméricos aplicados na proteção de matrizes cimentíceas foi estudada após imersão de filmes livres em soluções aquosas agressivas, utilizando-se de diferentes métodos: DMA Tan Delta, DMA E", DSC, e TMA ${ }^{[10]}$. O pesquisador obteve valores diferentes para cada método. Os valores de $\mathrm{Tg}$ obtidos pelo pico da curva Tan Delta superaram os valores obtidos pelos demais métodos. Os valores estimados por TMA resultaram consideravelmente inferiores aos obtidos por DMA-E" e DSC. Como a técnica DMA-E" não exige a secagem dos corpos de prova antes do ensaio, o autor concluiu que os resultados obtidos por DMA-E” eram os mais confiáveis.

\section{Experimental}

Os procedimentos adotados foram em conformidade com a ASTM G53. O experimento consistiu na exposição de materiais em laboratório aos agentes degradantes: luz, calor, vapor d'água, controlados de maneira que simulem, artificialmente, o ambiente natural. Entretanto, devido a esses fatores agirem na atmosfera de modo aleatório e imprevisível, não se pode esperar que o ensaio em laboratório reproduza fielmente as variações das intempéries naturais.

Basicamente o aparelho de laboratório para o ensaio de intemperismo artificial é composto por oito lâmpadas de radiação ultravioleta, sistema de aspersão de água, controle de temperatura e umidade, controle de programação do ciclo, e suporte dos corpos de prova. Estas partes são acopladas em uma câmara de madeira permitindo que o pesquisador tenha a facilidade de operação dos controles de ajuste, colocação dos corpos de prova e na manutenção do equipamento.

Foram empregadas lâmpadas com as seguintes características:

- lâmpadas fluorescentes de baixa pressão (vapor de mercúrio), com emissão de radiação ultravioleta;

- emissão de radiação altamente concentrada entre 320 e $390 \mathrm{~nm}$;

- a taxa de emissão UVB/UVA é de aproximadamente 1:200. 
Foram moldadas placas de filmes de resina poliuretana vegetal de dimensões $60 \times 290 \times 3 \mathrm{~mm}$, as quais foram colocadas em suportes de alumínio existentes na câmara e submetidas a ciclos alternados entre 4 horas de condensação e 4 horas de radiação ultravioleta. Com intervalos de diferentes dias de exposição, foram retiradas placas expostas na câmara para que as suas características mecânicas fossem avaliadas em função do tempo de exposição ao intemperismo artificial.

Para a moldagem das placas de poliuretana foi feita a mistura manual dos componentes pré-polímero e poliol fornecidos pelo Laboratório de Química Analítica e Tecnologia dos Polímeros do IQSC/USP e a seguir retirou-se o ar incorporado utilizando-se uma bomba a vácuo, a fim de evitar possíveis bolhas de ar.

As placas de filme livre foram curadas em ambiente climatizado $\left(25^{\circ} \mathrm{C}\right.$ e $75 \%$ de umidade relativa do ar) seguindo-se com a colocação no suporte de alumínio acoplado à câmara. Este suporte permite que o fluxo de vapor transcorra pela superfície das placas e que os raios ultravioletas incidam sobre as mesmas. Segundo a norma, as placas dispostas na área de exposição à radiação devem ser rotacionadas diariamente de modo a atenuar as diferenças de radiação em diferentes regiões.

O ensaio para a caracterização mecânica, após exposição na câmara de intemperismo artificial, foi realizado conforme a norma ASTM D 638M-96. A partir das placas retiradas da câmara, foram recortados os corpos de prova nas dimensões especificadas na norma, utilizando-se um molde. $\mathrm{O}$ ensaio dinâmico-mecânico (DMA) foi realizado para a obtenção das propriedades viscoelásticas da poliuretana vegetal, método largamente utilizado para o estudo de materiais viscoelásticos ${ }^{[11]}$. Os equipamentos utilizados foram o DMA 983 Du Pont, acoplado ao analisador térmico TA 9900, além de um software para calibração automática e análise de dados, existentes no laboratório de Química Analítica e Tecnologia de Polímeros do Instituto de Química da Universidade de São Paulo/São Carlos. Neste experimento, a amostra foi submetida a uma solicitação senoidal, amplitude $0,6 \mathrm{~mm}$, à freqüência de $1 \mathrm{~Hz}$, com variação de temperatura de $5{ }^{\circ} \mathrm{C} /$ minuto, na faixa de temperatura de $22^{\circ} \mathrm{C}$ até $140^{\circ} \mathrm{C}$.

\section{Resultados e Discussão}

Após o início do funcionamento da câmara de intemperismo artificial, foram retiradas duas placas, periodicamente, para a realização da caracterização mecânica. Foram obtidas placas confeccionadas com a poliuretana vegetal com os dias e os respectivos números de horas de exposição ao intemperismo artificial, conforme são explicitados na Tabela 1 (Tabela 1: Número de horas de exposição ao intemperismo artificial).

A exposição ao intemperismo artificial resultou em pequenas alterações nas propriedades mecânicas da resina poliuretana vegetal. Os resultados mostraram que:

- Conforme se observa na Figura 1, após 756 horas de exposição os valores de resistência à tração foram crescentes;
Tabela 1. Número de horas de exposição ao intemperismo artificial.

\begin{tabular}{ccc}
\hline Dias & $\begin{array}{c}\text { Tempo de permanência } \\
\text { na câmara (h) }\end{array}$ & $\begin{array}{c}\text { Exposição à radiação } \\
\text { ultravioleta (h) }\end{array}$ \\
\hline 18 & 432 & 216 \\
36 & 864 & 432 \\
63 & 1512 & 756 \\
127 & 3048 & 1524 \\
170 & 4080 & 2040 \\
218 & 5232 & 2616 \\
\hline
\end{tabular}

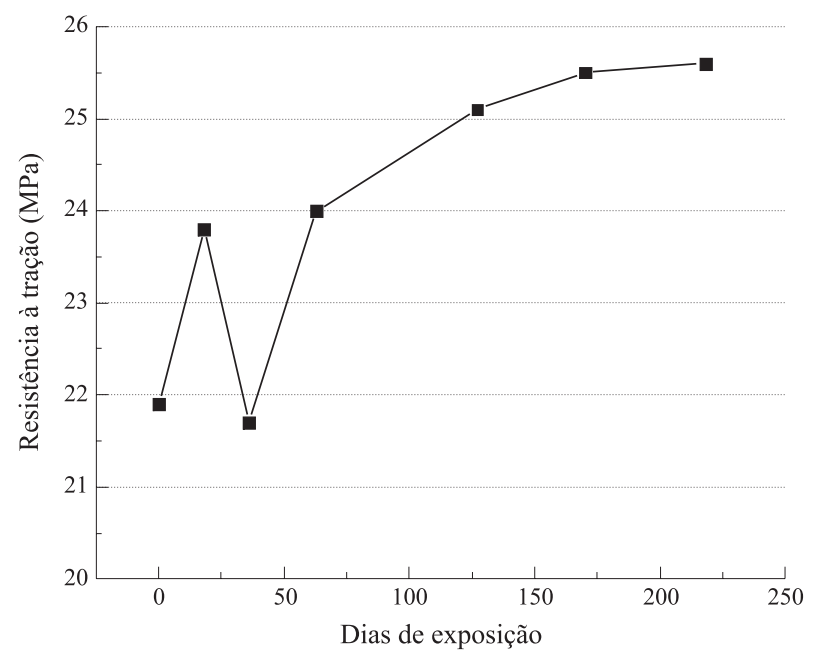

Figura 1. Resistência à tração das amostras de resina poliuretana vegetal.

- Os valores de tensão de ruptura são observados na Figura 3. Após 2626 horas de exposição nota-se o decréscimo da tensão $(25,3 \mathrm{MPa})$ em relação à data anterior $(26 \mathrm{MPa})$, mas ainda superior ao valor inicial (22 $\mathrm{MPa})$;

- $\mathrm{O}$ alongamento na ruptura mostrou ser uma propriedade mecânica com valores variados, descritos na Figura 4. Inicialmente, observa-se a diminuição de $29 \%$ para $16 \%$, com 432 horas de exposição. Após essa data, os valores mantiveram-se entre $19 \%$ e $17 \%$;

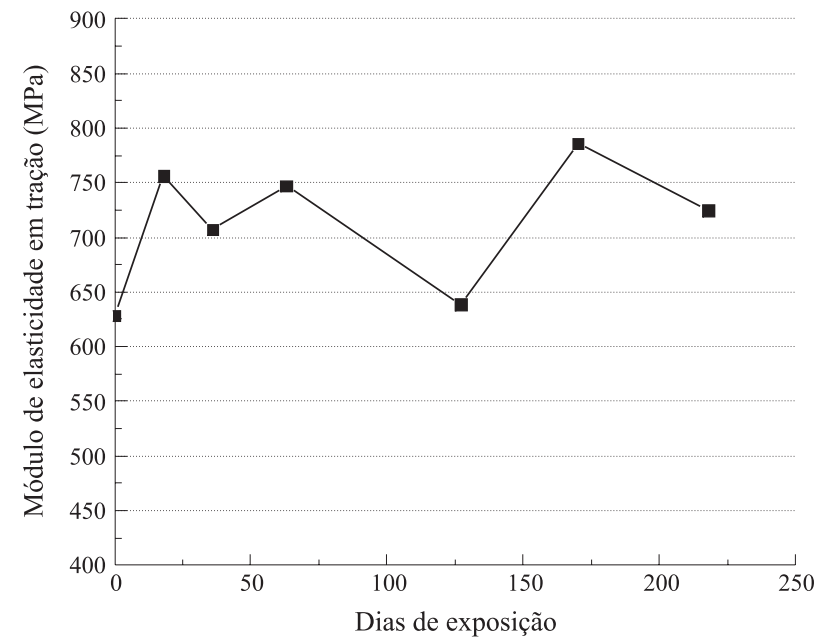

Figura 2. Módulo de elasticidade das amostras de resina poliuretana. 


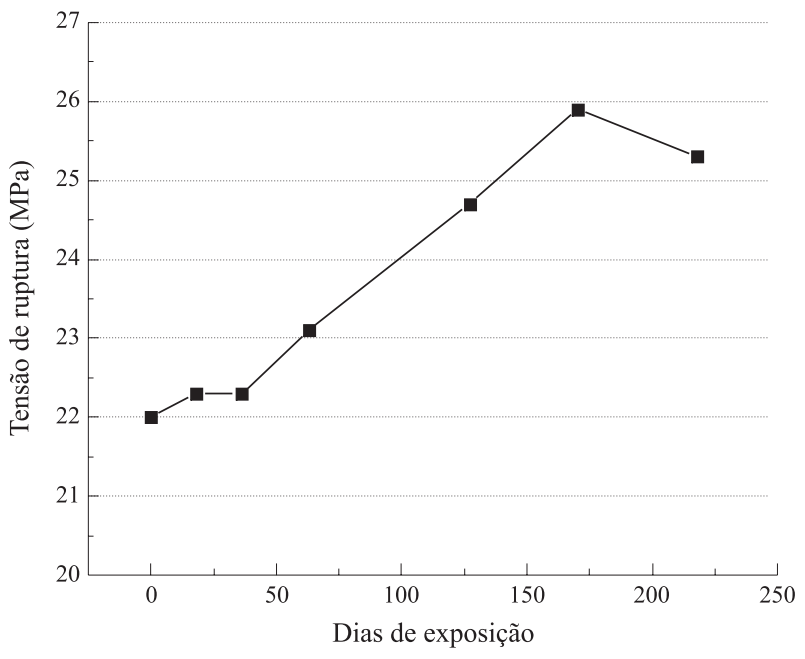

Figura 3. Tensão de ruptura das amostras de resina poliuretana.

- Os valores do módulo de elasticidade descritos na Figura 2 apresentaram valores entre $630 \mathrm{MPa}$ e $725 \mathrm{MPa}$, pois estão relacionados com os valores da deformação;

- Os valores de tensão de escoamento obtidos após a exposição foram maiores que o valor inicial (sem exposição ao intemperismo artificial). Entretanto, os valores apresentaram pequenas variações entre $20 \mathrm{MPa}$ e $24 \mathrm{MPa}$.

Dentre as propriedades mecânicas, o alongamento é a propriedade mais sensível para se avaliar efeitos na superfície do material devido a deformações que podem ocorrer no substrato. Verifica-se uma queda para $15 \%$ para maiores valores de exposição, o que é significativo, pois se o filme for mais fino (no caso da aplicação) essa queda pode ser mais drástica ainda. Esses resultados requerem a avaliação por um período maior de exposição, a fim de verificar se a redução do alongamento é crescente ou se ocorre a estabilização.

A análise dinâmico-mecânica (DMA) permitiu a obtenção de vários parâmetros, sendo um deles a relação entre o módulo de perda e o de armazenamento, conhecido como Tan Delta. As curvas do módulo de armazenamento, módulo de perda e Tan Delta em função da temperatura podem ser

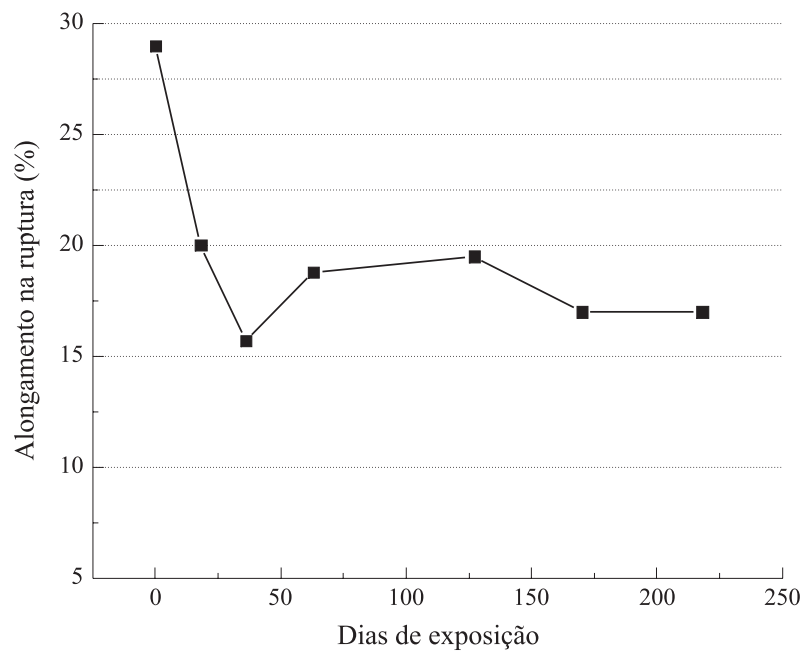

Figura 4. Deformação dos corpos-de-prova na ruptura, após exposição ao intemperismo artificial. observadas nas Figuras 5, 6 e 7, respectivamente, evidenciando que a exposição ao intemperismo artificial ocasionou pequenas variações nas propriedades tal como o enrijecimento devido a diminuição de Tan delta.

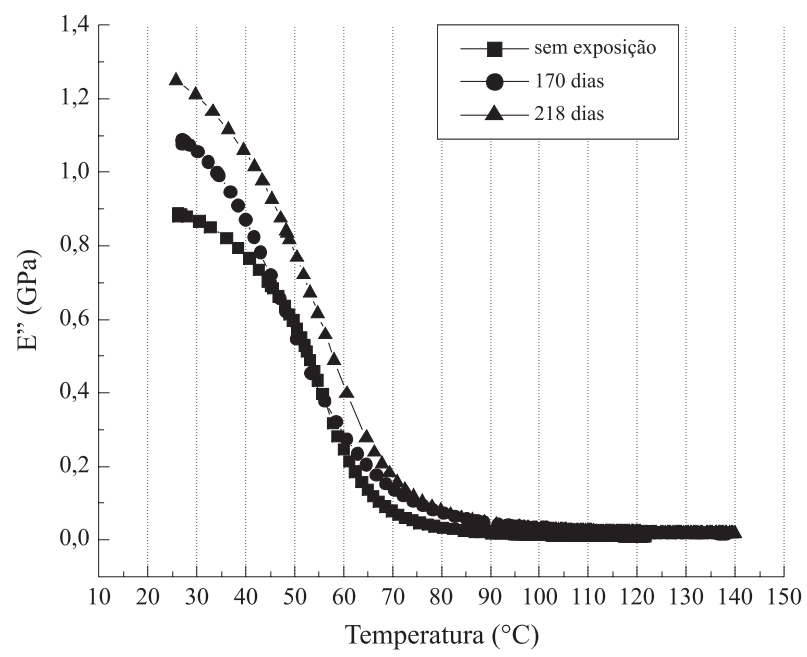

Figura 5. Curvas do módulo de armazenamento $\left(\mathrm{E}^{\prime}\right) v s$. temperatura, do sistema base poliuretano vegetal após exposição ao intemperismo artificial.

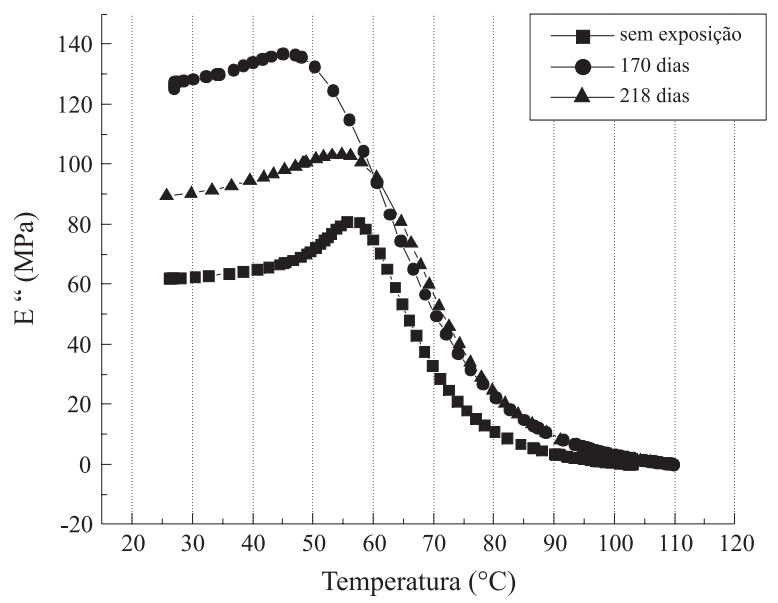

Figura 6. Curvas do módulo de perda $\left(\mathrm{E}^{\prime \prime}\right)$ vs. temperatura, do sistema base poliuretano vegetal após exposição ao intemperismo artificial.

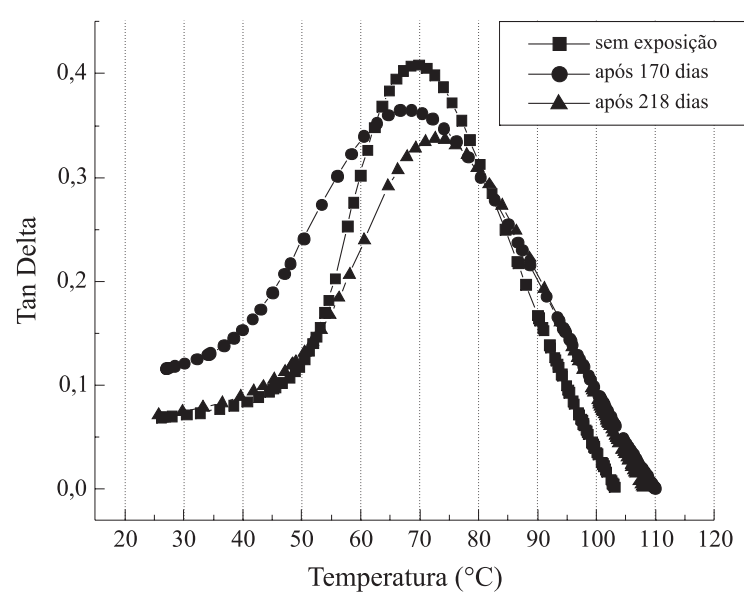

Figura 7. Curvas de tan delta ( $\tan \delta$ ) vs. temperatura, do sistema base poliuretano vegetal após exposição ao intemperismo artificial. 
Tabela 2. Valores máximos do módulo de perda.

\begin{tabular}{ccc}
\hline Dias de exposição & $\begin{array}{c}\text { Módulo de perda }\left(\mathbf{E}^{\prime \prime}\right) \\
(\mathbf{M P a})\end{array}$ & $\begin{array}{c}\text { Temperatura } \\
\left({ }^{\circ} \mathbf{C}\right)\end{array}$ \\
\hline 0 & 80,75 & 55,58 \\
170 & 136,8 & 45 \\
218 & 103,1 & 54,77 \\
\hline
\end{tabular}

Tabela 3. Valores máximos de Tan Delta.

\begin{tabular}{ccc}
\hline Dias de exposição & Tan Delta & $\begin{array}{c}\text { Temperatura } \\
\left({ }^{\circ} \mathbf{C}\right)\end{array}$ \\
\hline 0 & 0,408 & 69 \\
170 & 0,365 & 67,8 \\
218 & 0,337 & 72,6 \\
\hline
\end{tabular}

O máximo do pico da curva do módulo de perda corresponde à situação de máxima dissipação de energia mecânica, que na região de transição vítrea está associada à mudança do estado vítreo para o elástico.

As Tabelas 2 e 3 mostram que os valores de temperatura de transição vítrea considerando-se Tan Delta são maiores que os valores obtidos pelo máximo valor do módulo de perda, corroborando com estudos anteriores ${ }^{[10]}$. Desse modo, a temperatura de transição vítrea obtida pelo máximo módulo de perda para o sistema poliuretano vegetal sem exposição ao intemperismo artificial foi próxima ao valor obtido para o mesmo sistema com 217 dias de exposição.

Trabalhos realizados por pesquisadores ressaltam que os resultados de ensaios mecânicos e aqueles obtidos por experimentos dinâmico-mecânicos nem sempre estabelecem uma associação direta ${ }^{[12]}$. A relação entre essas propriedades é principalmente dependente da natureza do material em questão, não sendo possível se estabelecer uma regra simples para esta correlação.

\section{Conclusões}

Os resultados obtidos no ensaio de exposição ao intemperismo artificial mostram que o sistema poliuretano vegetal sofreu alterações devido a exposição ao intemperismo artificial, entretanto, estudos com maiores tempos de exposição são necessários para avaliar se essas alterações são capazes de ocasionar falhas no revestimento.

A determinação da temperatura de transição vítrea é importante para a avaliação do comportamento do revestimento nas condições de aplicação. Os valores de Tg não devem ser próximos da temperatura de serviço que pode sofrer alterações prejudicando o desempenho do polímero. A temperatura de transição vítrea encontrada foi próxima de $54^{\circ} \mathrm{C}$ para o sistema poliuretano analisado. Outro fator importante relacionado com a determinação da temperatura de transição vítrea é evitar os riscos de que o sistema de proteção entre em contato com líquidos ou sólidos a altas temperaturas, quando aplicado na indústria química.

\section{Referências Bibliográficas}

\section{AMERICAN SOCIETY FOR TESTING AND} MATERIALS - ASTM G - 53 Operating Light - and Water-Exposure Apparatus (Fluorescent UVCondensation Type) for Exposure of Nonmetallic Materials.

2. AMERICAN SOCIETY FOR TESTING AND MATERIALS - ASTM D - 638M - 96 Standard Test Method for Tensile Properties of Plastics (Metric).

3. Araújo, L. C. R. - "Caracterização Química, Térmica e Mecânica de Poliuretanas Elastoméricas Baseadas em Materiais Oleoquímicos”, Dissertação de Mestrado, Universidade de São Paulo, Brasil (1992).

4. Almeida, A. E. F. de S. - "Desempenho de revestimento poliuretano vegetal, frente a outros polímeros, quanto a proteção do concreto em ambientes agressivos", Dissertação de Mestrado, Universidade de São Paulo, Brasil (2000).

5. Feldman, D. - "Polymeric Building Materials", Elsevier Applied Science (1989).

6. Parker, D. H. - Principles of Surface Coating Technology. Intersciense Publishers (1965).

7. Grassie, N. \& Scott, G. - "Polymer Degradation and Stabilization", Cambridge University Press (1985).

8. Chatfield, H. W. - "The Science of Surface Coatings", Ernest Benn Limited, London (1962).

9. Sperling, L. H. - "Introdution to Physical Polymer Science", Wiley- Interscience Publication (1992).

10. Repette, W. L. - "Modelo de previsão de vida útil de revestimentos de proteção da superfície do concreto em meios de elevada agressividade", Tese de Doutorado, Universidade de São Paulo (1997).

11. Rosen, S. L. - Fundamental Principles of Polymeric Materials, Wiley - Interscience Publication (1982).

12. Cassu, S. N. \& Felisberti, M. I. - Quim. Nova, Vol. 28, n. 2, p. 255-263 (2005).

Enviado: 23/01/06

Reenviado: 12/04/06

Aprovado: 03/05/06 